\title{
TRP Channels Expression Profile in Human End-Stage Heart Failure
}

\author{
Martin Dragún ${ }^{1}$, Andrea Gažová ${ }^{2}$, Ján Kyselovič ${ }^{3}$, Michal Hulman ${ }^{4}$ and Marek Mát uš ${ }^{1, *}$ (D) \\ 1 Department of Pharmacology and Toxicology, Faculty of Pharmacy, Comenius University in Bratislava, \\ Odbojarov 10, 83232 Bratislava, Slovakia \\ 2 Institute of Pharmacology and Clinical Pharmacology, Faculty of Medicine, Comenius University in \\ Bratislava, Spitalska 24, 81372 Bratislava, Slovakia \\ 3 5th Department of Internal Medicine, University Hospital Bratislava-Ružinov, Ruzinovska 6, \\ 82606 Bratislava, Slovakia \\ 4 Clinic of cardiac surgery, National Institute for Cardiovascular Diseases, Pod Krasnou Horkov 1, \\ 83348 Bratislava, Slovakia \\ * Correspondence: matus@fpharm.uniba.sk
}

Received: 17 May 2019; Accepted: 11 July 2019; Published: 16 July 2019

\begin{abstract}
Objectives: Many studies indicate the involvement of transient receptor potential (TRP) channels in the development of heart hypertrophy. However, the data is often conflicted and has originated in animal models. Here, we provide systematic analysis of TRP channels expression in human failing myocardium. Methods and results: Left-ventricular tissue samples were isolated from explanted hearts of NYHA III-IV patients undergoing heart transplants $(n=43)$. Quantitative real-time PCR was performed to assess the mRNA levels of TRPC, TRPM and TRPV channels. Analysis of functional, clinical and biochemical data was used to confirm an end-stage heart failure diagnosis. Compared to myocardium samples from healthy donor hearts $(n=5)$, we detected a distinct increase in the expression of TRPC1, TRPC5, TRPM4 and TRPM7, and decreased expression of TRPC 4 and TRPV2. These changes were not dependent on gender, clinical or biochemical parameters, nor functional parameters of the heart. We detected, however, a significant correlation of TRPC1 and MEF2c expression. Conclusions: The end-stage heart failure displays distinct expressional changes of TRP channels. Our findings provide a systematic description of TRP channel expression in human heart failure. The results highlight the complex interplay between TRP channels and the need for deeper analysis of early stages of hypertrophy and heart failure development.
\end{abstract}

Keywords: heart failure; human; TRP channels; calcium signaling

\section{Introduction}

Heart failure (HF) is a culmination of different pathophysiological conditions on heart muscle which cause progressive worsening of heart function [1]. HF is usually preceded by chronic cardiac stress that could be the result of a cardiac injury (e.g., myocardial infarct, hypertension, valvular heart disease) and leads to structural changes, such as left ventricle hypertrophy, to maintain physiologic heart output [2,3]. Functionally, HF is preceded by impaired calcium homeostasis-Contraction and $\mathrm{Ca}^{2+}$ transients are markedly prolonged and restoration of low diastolic $\mathrm{Ca}^{2+}$ concentrations is impaired [4]. Increased $\mathrm{Ca}^{2+}$ levels during diastole can be related to depressed protein expression levels of sarco/endoplasmic reticulum $\mathrm{Ca}^{2+}$-ATPase (SERCA) or decreased rates of sarcoplasmic reticulum (SR) $\mathrm{Ca}^{2+}$ uptake [5]. After depletion of internal $\mathrm{Ca}^{2+}$ stores, the store-operated calcium entry (SOCE) is activated [6]. Multiple proteins and channels are involved in SOCE, e.g., STIM proteins, Orai channels or channels from the transient receptor potential (TRP) family. 
TRP channels are a family of non-voltage dependent calcium-permeable ion channels first discovered in Drosophila [7]. The mammalian TRP family represents a big group of 28 cation channels divided according their structural homology into six subfamilies: TRP canonical (TRPC), TRP melastin (TRPM), TRP vanniloid (TRPV), TRP ankyrin (TRPA), TRP mucolipin (TRPML) TRP polycystin (TRPP) [8]. TRP can be activated by various stimuli, such as thermally-activated channels (TRPV, TRPM or TRPA) or TRPC activated by phospholipase C (PLC) [9]. There are also differences inside the groups in regulation of the channel activity e.g., TRPC subfamily, where TRPC 3 and TRPC 6 can be activated by stretch [9], TRPC 1 and TRPC 3 by SOCE [10], TRPC4 and TRPC 5 are regulated by G protein-coupled receptor [11] and TRPC6 and TRPC7 are regulated by $\mathrm{Ca}^{2+}$ in multiple ways [12].

The physiological function of TRP channels in the heart is still under debate. Inhibition of TRP channels did not cause any defects under physiological conditions, but they seem to play a critical role in pathophysiology of numerous heart diseases [13]. TRP channels most often linked with hypertrophy and HF are from the TRPC subfamily. There are multiple animal and cell culture models of hypertrophy and HF confirming their role in these pathologies: knockout of Trpc genes or use of TRPC inhibitors prevents hypertrophy and HF development, and their increased expressions was detected after neuroendocrine agonist infusion (e.g., endothelin-1, phenylephrine and angiotensin II) and/or pressure overload stimulation $[13,14]$. It appears that they act through activation of calcineurin/nuclear factor of activated T cells (NFAT) signaling pathway, which leads to the activation of the pro-hypertrophic gene expression. Additionally, it has been shown that calcineurin/NFAT cascade can positively regulate the activity of TRPC channels $[15,16]$.

In addition to TRPC channels, some members of TRPM and TRPV channels are also involved in hypertrophy and HF development. Inhibition of TRPV2 accumulation at sarcolemma improves the survival of animals with HF, prevents fibrosis and ventricular dilation and decreases the activation of calmodulin-dependent protein kinase II [17]. Moreover, disruption of the Trpv1 gene can prevent the progression of cardiac hypertrophy [18].

However, the data on the role of TRP channels in the human HF is less complete or anecdotal. To our knowledge, only three studies have touched this topic in systematic manner $[15,19,20]$. The aim of this study was to investigate the gene expression of channels from the TRPC, TRPM and TRPV families in human heart and their possible expression changes in human HF compared to a non-failing healthy myocardium.

\section{Materials and Methods}

\subsection{Human Heart Samples}

For this study, 43 patients were chosen who were diagnosed with heart failure in NYHA III-IV class (HF group). These patients were indicated for heart transplant surgery and underwent the surgery in National Institute for Cardiovascular Diseases from August 2009 to June 2013. The samples of heart tissue were isolated from explanted left ventricle without epicardial fat, as a cross-section of the whole wall of the ventricle. Tissue samples of non-failing controls $(C O N ; n=5)$ were obtained from donor hearts that were prepared for transplantation, but the surgery was not performed. Clinical and biochemical data were obtained from patient documentation and were not older than 3-6 months before transplantation, except the heart weight which was measured after the surgery. Because the clinical data was not available from all patients, the number of data points for statistical evaluation can differ across the data groups and is noted in respective tables. The whole project and experimental procedures were approved by the Ethics committee of the National Institute for Cardiovascular Diseases in Bratislava, Slovak republic (No. IK-NP-0021-24/1426/14 and NUSCH EK 126/180509 from 11 November 2008). The study conformed to the principles outlined in the Declaration of Helsinki, all patients (or their legal representatives) were informed about and gave informed consent prior to participation in this project. 


\subsection{RNA Isolation and Real-Time Polymerase Chain Reaction}

Total RNA was isolated from left ventricle samples using Tri-Reagent ${ }^{\circledR}$ (Sigma-Aldrich, St. Louis, MO, USA) according to the recommended manufacturer's protocol. The quality of isolated RNA was verified with electrophoresis on $2 \%$ agarose gel. Quality and quantity of the RNA was measured with spectrophotometric analysis (NanoDropND-1000, Thermo Scientific, Waltham, MA, USA). Reverse transcription was performed using High-Capacity cDNA KIT with RNAse inhibitor (Applied Biosystems, Foster City, CA, USA). SYBR Green detection (SYBR Select Master Mix, Life Technologies, Carlsbad, CA, USA) was used to perform quantitative RT-PCR on StepOnePlus ${ }^{\circledR}$ Real-Time PCR System (Life Technologies) according to manufacturer's instructions. Primers (Sigma-Aldrich) used for amplification of genes of interest are listed in Table S3. All primers were verified to yield a single PCR product with the correct molecular weight, and the absence of signal was verified when reverse transcription was omitted. The results were normalized to the expressions of endogenous reference genes (HPRT1, hypoxanthine phosphoribosyltransferase 1; B2M, beta-2-microglobulin). Experimental data can be found in Table S4.

\subsection{Statistical Analysis}

PCR efficacy and quantification cycle values for each sample were determined with LinRegPCR software (version 2017.1). Shapiro-Wilk test was used as normality test. To compare two groups with normally distributed data, Student's $t$-test was used. For non-normally distributed data, we performed the Mann-Whitney test. ANOVA was used to assess the differences among multiple groups. Statistical correlation between two data groups was determined by Pearson correlation coefficients. All data were handled by GraphPad Prism (GraphPad Software Inc., San Diego, CA, USA, version 6). All data are presented as average \pm standard error of the mean.

\section{Results}

\subsection{Clinical and Biochemical Characteristics of Patients with Heart Failure}

The wet weight of the explanted hearts was higher than the physiologic range (250-350 g) and this was observed both in the sex-specific group of women and men. The diagnosed HF was in last stages as shown by the left ventricle ejection fraction (LVEF) that was decreased to $22.64 \pm 1.407 \%$. The failing hearts of women displayed higher ejection fraction than male hearts. Both average Left ventricle end-diastolic diameter (LVEDD) and right ventricle end-diastolic diameter (RVEDD) was increased. QT interval values were within the physiological range $(<0.44 \mathrm{~s})$ and QRS complex values were slightly elevated (Table 1).

The average age of patients was 50.84 years, where women were insignificantly younger than men. The patients' average BMI fell within the overweight range. Blood pressure values were within the physiological limits, although the heart rate was elevated (Table 1).

Table 1 also summarizes the biochemical parameters of the patient group. Plasmatic concentrations of N-terminal pro B-type natriuretic peptide (NT-proBNP) and troponin T were in the range of values that confirms end-stage HF. We detected no sex-specific differences in these concentrations. Levels of cholesterol and triacylglyceride (TAG) were not increased, possibly due to antihyperlipidemic therapy.

Table 1. Patients' clinical characteristics.

\begin{tabular}{cccc}
\hline & ALL & Female & Male \\
\hline HW [g] & $635.8 \pm 24.09(30)$ & $415.8 \pm 43.04(4)$ & $669.7 \pm 19.97 \#(26)$ \\
LVEF [\%] & $22.64 \pm 1.407(42)$ & $30.88 \pm 5.363(8)$ & $20.71 \pm 1.003(34)$ \\
LVEDD [mm] & $69.35 \pm 1.750(40)$ & $54.50 \pm 3.718(8)$ & $73.06 \pm 1.346 \#(32)$ \\
RVEDD [mm] & $33.87 \pm 0.7404(39)$ & $32.25 \pm 1.146(8)$ & $34.29 \pm 0.8752(31)$ \\
QRS [s] & $0.1372 \pm 0.006557(36)$ & $0.1420 \pm 0.01718(7)$ & $0.1361 \pm 0.007155(27)$ \\
\hline
\end{tabular}


Table 1. Cont

\begin{tabular}{cccc}
\hline & ALL & Female & Male \\
\hline QT [s] & $0,4221 \pm 0.00722(34)$ & $0.4263 \pm 0.01225(7)$ & $0.4210 \pm 0.008612(27)$ \\
Age [year] & $50.84 \pm 1.281(43)$ & $46.88 \pm 2.553(8)$ & $51.74 \pm 1.433(35)$ \\
BMI [kg/m $\left.{ }^{2}\right]$ & $27.11 \pm 0.6984(42)$ & $24.81 \pm 1.797(8)$ & $27.65 \pm 0.7356 \#(34)$ \\
SBP [mmHg] & $109.3 \pm 1.964(42)$ & $103.8 \pm 5.775(8)$ & $110.6 \pm 1.996(34)$ \\
DBP [mmHg] & $70.93 \pm 1.194(42)$ & $69.00 \pm 2.790(8)$ & $71.38 \pm 1.330(34)$ \\
HR [min $\left.{ }^{-1}\right]$ & $79.95 \pm 1.754(43)$ & $78.88 \pm 2.689(8)$ & $80.21 \pm 2.085(34)$ \\
NT-proBNP [ng/l] & $4666 \pm 631.5(40)$ & $5132 \pm 1152(8)$ & $4549 \pm 741.7(32)$ \\
Troponin T [ng/l] & $30.18 \pm 5.202(31)$ & $38.53 \pm 17.99(6)$ & $28.09 \pm 4.911(25)$ \\
Cholesterol [mmol/l] & $4.133 \pm 0.1892(32)$ & $4.528 \pm 0.3372(5)$ & $4.059 \pm 0.2145(27)$ \\
TAG [mmol/l] & $1.684 \pm 0.2430(31)$ & $1.095 \pm 0.1395(6)$ & $1.825 \pm 0.2936 \#(25)$
\end{tabular}

HW-Heart weight, LVEF-Left ventricle ejection fraction, LVEDD—Left ventricle end-diastolic diameter, RVEDD-Right ventricle end-diastolic diameter, BMI-Body mass index, SBP-Systolic blood pressure, DBP-Diastolic blood pressure, HR-Heart rate, NT-proBNP-N-terminal pro B-type natriuretic peptide and TAG-triacylglyceride blood concentrations. Number of patients where data was available shown in brackets; Student's $t$-test: $\# p<0,05$ vs. F.

\subsection{Expression of TRPC Channels}

We detected the expression of TRPC $1,-3,-4,-5$ and -6 channels, whereas TRPC7 was not detectable (Figure 1). The expression of $\operatorname{Trpc} 1$ gene was increased by more than $50 \%$ in the failing myocardium. The strongest elevation in expression, 3.8 times higher in failing hearts compared to CON, was observed in TRPC 5 channel. Gene expression of TRPC 4 channel was downregulated by $60 \%$. There was no change between the groups in TRPC 3 and TRPC 6 channels expression.

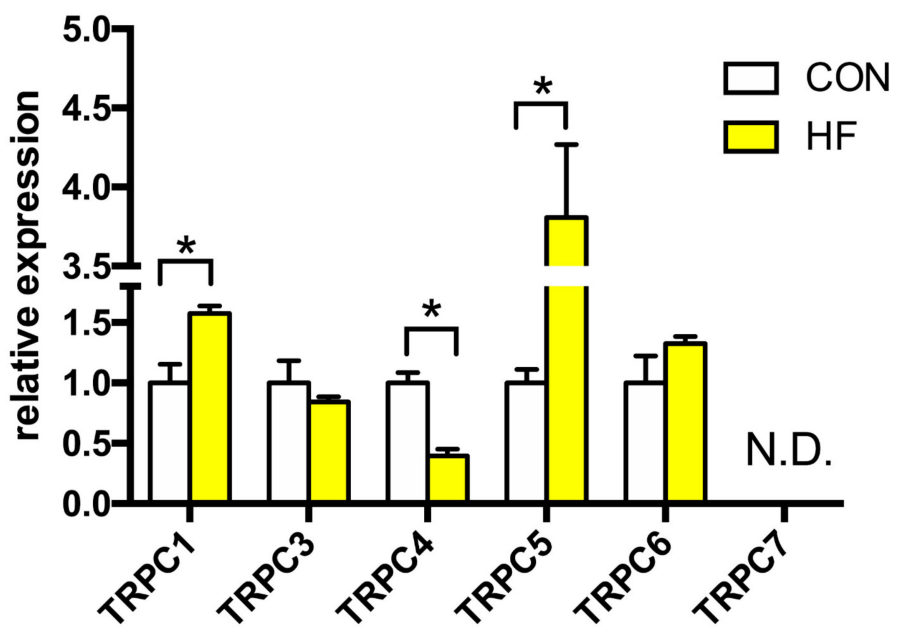

Figure 1. Relative gene expression of TRPC channels in the left ventricle of patients with heart failure $(\mathrm{HF}, \mathrm{n}=43)$ and non-failing $(\mathrm{CON}, \mathrm{n}=5)$ controls. N.D.-Non-detectable. Student's $t$-test: ${ }^{*} p<0.05$ vs. CON.

\subsection{Expression of TRPM Channels}

To this date, to our knowledge there are no information that links the TRPM channels subfamily and human HF, and only sparse data is available about their expression in human heart in general. We studied the expression profile of TRPM2, $-4,-6$ and -7 (Figure 2). The expression of TRPM2 channel was not altered in HF, however, we detected significantly increased expression of TRPM4 (more than 65\% compared to CON) and TRPM7 channels (about 170\% of CON). TRPM6 channel expression was not detected in either $\mathrm{CON}$ or failing myocardium. 


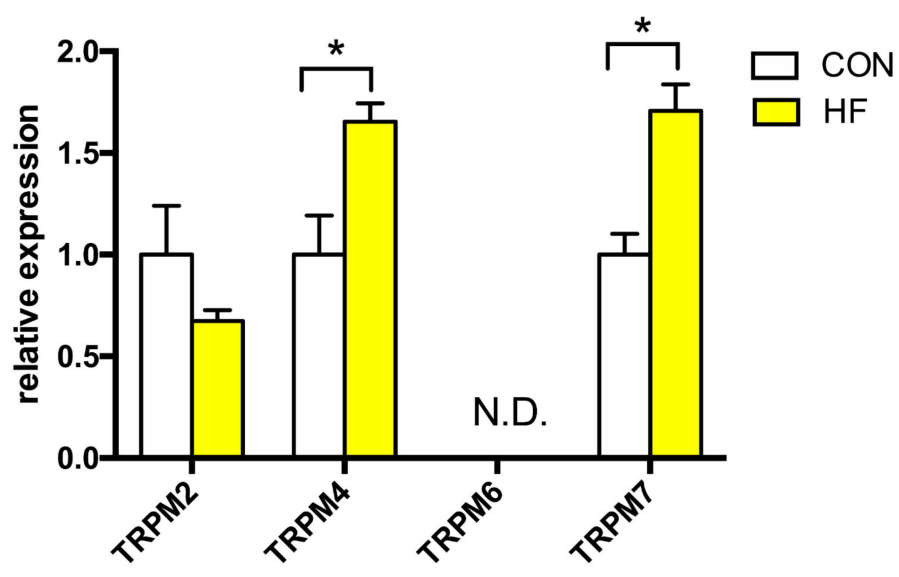

Figure 2. Relative gene expression of TRPM channels in the left ventricle of patients with heart failure $(\mathrm{HF}, \mathrm{n}=43)$ and non-failing $(\mathrm{CON}, \mathrm{n}=5)$ controls. N.D.-Non-detectable. Student's $t$-test: ${ }^{*} p<0.05$ vs. CON.

\subsection{Expression of TRPV Channels}

Analysis of the mRNA expression levels of TRPV1 and TRPV2 channels is summarized in Figure 3. We discovered significant decrease in gene expression levels of TRPV2. The average expression of TRPV1 displayed a tendency toward an increase in the HF group; however, this effect did not reach statistical significance.

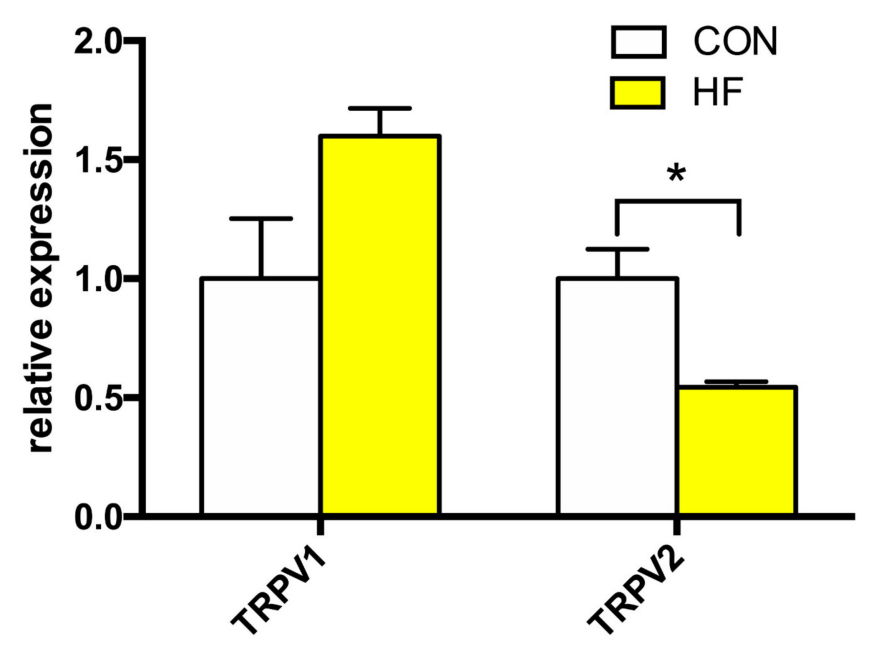

Figure 3. Relative gene expression of TRPV channels in the left ventricle of patients with heart failure $(\mathrm{HF}, \mathrm{n}=43)$ and non-failing $(\mathrm{CON}, \mathrm{n}=5)$ controls. N.D.-Non-detectable. Student's $t$-test: ${ }^{*} p<0.05$ vs. CON.

\subsection{The Expression of TRPC1 Correlates with MEF2c Gene Expression}

As showed above, the expression of several TRP channels was significantly changed in failing myocardium. Because the alterations to $\mathrm{Ca}^{2+}$ homeostasis could affect some $\mathrm{Ca}^{2+}$-dependent transcription factors, we also analyzed the expression of MEF2a, MEF2c and NFAT3. While we detected no significant changes in the expression of MEF2a or NFAT3 (Table S1), MEF2c expression was significantly elevated by more than $70 \%$ and its expression positively correlated with $\operatorname{Trpc1}$ expression (Figure 4). 

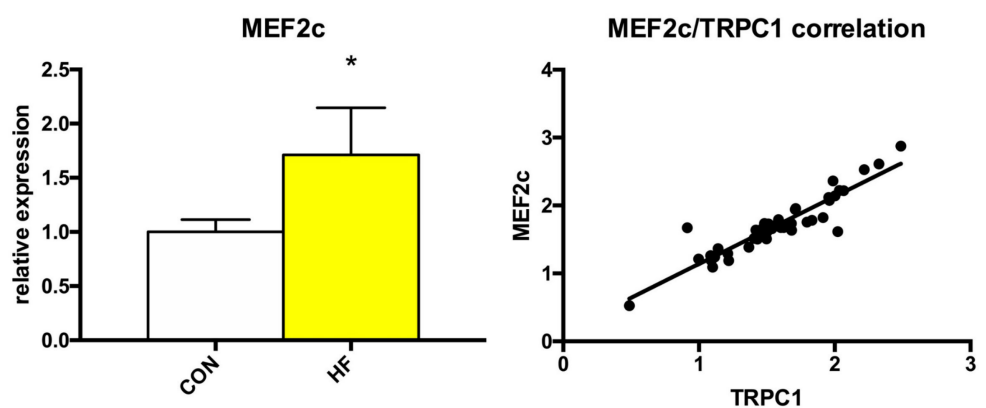

Figure 4. Relative gene expression of MEF2c and positive correlation between MEF2c and TRPC1 relative expressions. Student's $t$-test: ${ }^{*} p<0.05$ vs. CON; Pearson $\mathrm{R}^{2}=0.8487$.

\subsection{Clinical Status and TRP Expression}

To better characterize the role of individual TRP channel isoforms in and their connection to different pathophysiological mechanisms of HF, we analyzed the gene expressions in regard to different subgroups or correlation with different parameters. Interestingly, we did not detect any significant differences in TRP expression between male and female HF patients, nor between HF based on ischemic or non-ischemic background. Furthermore, the Trp gene expression was not influenced by (i.e., no significant correlation was detected with) either functional heart parameters (QT, QRS duration, LVEF), clinical parameters (age, BMI, SBP, DBP or HR), nor biochemical data (NT-proBNP, troponin T levels, total cholesterol or TAG) (Table S2).

\section{Discussion}

HF is a complex syndrome defined as a progressive loss of heart's blood-pumping function. HF invariably leads to cardiac hypertrophy and progressive disruption of calcium homeostasis $[3,4]$. TRP channels are non-voltage dependent, calcium-permeable cation channels that have been shown to play a critical role in HF and myocardial hypertrophy development [14]. However, there is limited data about their expression in a failing human heart. In this study, we focused on determining the gene expression changes of channels from TRPC, TRPM and TRPV subfamily in human HF compared to non-failing healthy controls.

\subsection{Clinical and Biochemical Data}

Clinical and biochemical data received from outpatient documentation showed severe heart damage. According to National Institute for Health and Care Excellence, patients with NT-proBNP, one of the main clinical diagnostic biomarkers of HF, levels above $2000 \mathrm{ng} / \mathrm{L}$ are suspected for HF and need echocardiographic examination [21]. Average NT-proBNP and troponin C levels of HF patients included in this study were significantly increased. Together with the very low average LVEF these findings confirm the end-stage of HF. HF patients had much higher heart weight than the expected physiological values, probably due to cardiac hypertrophy development. As expected, in the male sex-specific group, the heart weight was significantly higher compared to women.

Interestingly, we detected no significant correlation between either clinical, functional or biochemical parameters of HF patients and the expression of followed genes. This might be explained by the end-stage of the heart failing (requiring heart transplant due to compensatory mechanisms reaching their limits). This would require follow-up of this question in earlier stages of HF.

\subsection{TRPC Channels}

TRPC channels are divided according their sequence homology and function into two subgroups: TRPC3/6/7 and TRPC1/4/5; TRPC2 is a pseudogene in humans [22]. The critical role of TRPC in cardiac hypertrophy and HF was shown in multiple animal models. In models of pressure overload induced- and norepinephrine induced hypertrophy, mouse lacking Trpc1 gene were protected against 
hypertrophy development [23]. Although Bush et al. did not detect any change in gene expression of TRPC1 in human failing heart, Morine et al. showed significant increase in Trpc1 gene expression, in agreement with our results $[15,20]$. Increased expression of TRPC1 channel can result in higher SOCE into the cardiomyocyte, where this calcium influx could activate several prohypertrophic signaling pathways [24]. Also, we found that TRPC1 gene expression strongly correlates with expression of the MEF2c transcription factor. This could present one of mechanisms of gradual decay in the cardiomyocyte function, even though it was shown in animal models that TRPC-dependent $\mathrm{Ca}^{2+}$ current activates predominantly the calcineurin/NFAT pathway [16]. In our study, gene expression of TRPC4 channel was significantly decreased, which is controversial, because transgenic mice overexpressing dominant negative TRPC4 mouse showed significantly reduced cardiac hypertrophy after transverse aortic constriction [25]. In previous studies on human HF patients, TRPC4 channel gene expression was increased [20] or not changed [15]. This differences in reported Trpc4 gene expression could be caused by different heterotetramers that TRPC4 can form [26] or the etiology of HF. Last member of TRPC family whose expression was significantly changed is TRPC5 channel. Although the TRPC 5 channel was not detected in two previous studies, we found almost four times higher gene expression compared to the healthy group. TRPC5 is a redox sensitive channel. Oxidized phospholipids can activate this channel and cause acute stimulatory effects on $\mathrm{Ca}^{2+}$-entry that can be inhibited by TRPC 5 antibody or dominant-negative mutant TRPC5 [27]. Nevertheless, TRPC5 is not often linked with cardiac hypertrophy or HF and more data is needed for elucidating its function in this pathology. Interestingly, members of TRPC family that are most under debate in association with hypertrophy and HF, TRPC3 and TRPC6, were not significantly changed in human setting. Interestingly, the stretch-activated TRPC6 seems to be also the key component in stress-stimulated contractility [28], possibly due to an increase in SR calcium load. This provides another $\mathrm{Ca}^{2+}$-dependent regulatory mechanism (independent of NFAT activation), which may be important in HF.

\subsection{TRPM Channels}

The TRPM subfamily contains eight isoforms. Selectivity to $\mathrm{Ca}^{2+}$ is different among them-TRPM7 is most selective for $\mathrm{Ca}^{2+}$ while TRPM4 and TRPM5 are virtually impermeable for $\mathrm{Ca}^{2+}$, although these two channels are $\mathrm{Ca}^{2+}$-activated [29-31]. To this date, the role of TRPM channels in human $\mathrm{HF}$ is unclear. To our knowledge, there is only one study that has investigated mRNA expression levels of TRPM channels in human HF. Morine et al. reported that the gene expressions of TRPM2, -3 and -8 were reduced in failing left ventricle (LV) and TRPM1, -6 and -7 were not detectable [20]. Our results showed increased gene expression of TRPM 4 and -7 and a trend towards reduction of TRPM2 mRNA expression. TRPM6 channel was not detected. Increased mRNA expression of TRPM4 was detected in spontaneously hypertensive rats, a model of cardiac hypertrophy [32]. TRPM4 channel is $\mathrm{Ca}^{2+}$-activated, $\mathrm{Na}^{+}$- and $\mathrm{K}^{+}$-permeable and its increased expression can probably participate in development of delayed after-depolarizations (DADs) during cardiac hypertrophy [33]. We hypothesize that TRPM4 could participate in arrhythmia development during HF, but there is a need for further studies to confirm this hypothesis, because so far, the $\mathrm{Ca}^{2}+$-dependent transient inward current that is necessary for DADs development was detected only in human atrial cells and not in ventricular cells [34]. The role of TRPM4 in cardiac hypertrophy is controversial and it seems to work as a negative regulator of cardiac hypertrophy development after angiotensin II infusion [35]. Increased expression of TRPM7 during HF could result in fibrogenesis in LV. $\mathrm{Ca}^{2+}$ currents through TRPM7 in human atrial fibroblasts can mediate fibrogenesis together with TGF- $\beta 1$ [36].

\subsection{TRPV Channels}

In this study, we analyzed the mRNA expression of two members of TRPV family-TRPV1 and TRPV2. Trpv1 gene expression was not changed and gene expression of Trpv2 was significantly reduced in HF. The TRPV2 channel is $\mathrm{Ca}^{2+}$-permeable channel and under basal conditions, it is localized both intracellularly [37] and at the intercalated disc [17]. Translocation to plasma membrane occurs after 
activation by several receptor agonists, such as insulin-like growth factor or platelet-derived growth factor, or by mechanical stress $[37,38]$. The role of TRPV2 channel in maintaining a normal cardiac function in healthy hearts and HF development is different. On the one hand, temporary elimination of TRPV2 in the normal heart results in disruption of intercalated disc structure and in decline of the heart function [39]. On the other hand, the inhibition of TRPV2 translocation to the plasma membrane improved myocardial dysfunction, improved survival of mice with dilated cardiomyopathy and slowed the progression of dilated cardiomyopathy [17]. In addition, in previous study, the Trpv2 gene expression was increased in left ventricle in human HF [20] which indicates a more complex role of TRPV2 channel in human HF.

The body of data about involvement of TRP channels in the pathophysiology of HF is growing rapidly. However, published results from animal models or human samples are controversial and many important questions remain unresolved. The common theme seems to be a complex regulation and functional interplay between TRP isoforms. The interpretation of such data is further complicated by the fact that TRPs undergo different post-translational modifications, including phosphorylation, $N$-glycosylation or polyester modification or covalent modifications of cysteine residues.

\subsection{Study Limitations}

The main limitation of the study is the reliance on mRNA levels to assess the expressional patterns of TRP genes. The results presented here need to be expanded with data on protein expressions, posttranslational modifications, and function, which should provide deeper insight into the role of TRP in HF. Another limitation are generally low expression levels of genes of interest, both in CON and HF group. This contributes to a relatively high variability, which is already inherently high in clinical setting. This makes the interpretation of the correlation analysis particularly complicated and a targeted investigation should be performed in the near future. The same complication extends to the TRPC1/MEF2c interplay. Even though a similar relationship between TRPC1 and MEF2 was observed in skeletal muscle [40], a targeted approach to study this possible mechanism in cardiac tissue is warranted and required. Finally, end-stage HF represents a time point, where most of the damage to the heart is done, and similar analysis of earlier stages of human HF would help to identify the underlying pathophysiological mechanisms.

\section{Conclusions}

In conclusion, TRP channels play a key role in human HF development and underlie a complex, but distinct changes in their expression which often differs from that observed in animal models. We suggest that there is a crucial role of TRPC (TRPC1, TRPC4 and TRPC5), TRPM4 and -7, and TRPV2 channels. We also submit that if TRP channels should present a feasible pharmacological target for patients with $\mathrm{HF}$, there is a need for investigation to further elucidate the role of TRP channels in man, and to investigate whether TRP channels are potential pharmacological targets in patients with HF.

Supplementary Materials: The following are available online at http://www.mdpi.com/1010-660X/55/7/380/s1, Table S1: Relative expression of MEF2a, MEF2c and NFAT3; Table S2: Correlation analysis; Table S3: Primer sequences; Table S4: Experimental data.

Author Contributions: Conceptualization, M.M. and J.K.; Methodology, M.M. and M.D.; Investigation, M.D. and A.G.; Formal Analysis, M.D. and M.M.; Supervision, M.M. and M.H.; Writing-Original Draft Preparation, M.D.; Writing-Review and Editing, M.M.; Funding Acquisition, J.K.

Funding: This research was supported by The Slovak Research and Development Agency [grant number APVV-14-0416]; The Ministry of Education, Science, Research and Sport of the Slovak Republic [grant number VEGA-1/0949/15]; and The Comenius University in Bratislava [grant numbers UK/201/2017; FaFUK/29/2017].

Conflicts of Interest: The authors declare no conflict of interest. 


\section{References}

1. Zhu, S.; Han, Z.; Luo, Y.; Chen, Y.; Zeng, Q.; Wu, X.; Yuan, W. Molecular mechanisms of heart failure: Insights from Drosophila. Heart Fail. Rev. 2017, 22, 91-98. [CrossRef] [PubMed]

2. Grossman, W.; Jones, D.; McLaurin, L.P. Wall stress and patterns of hypertrophy in the human left ventricle. J. Clin. Investig. 1975, 56, 56-64. [CrossRef] [PubMed]

3. Tham, Y.K.; Bernardo, B.C.; Ooi, J.Y.Y.; Weeks, K.L.; McMullen, J.R. Pathophysiology of cardiac hypertrophy and heart failure: Signaling pathways and novel therapeutic targets. Arch. Toxicol. 2015, 89, 1401-1438. [CrossRef] [PubMed]

4. Morgan, J.P.; Erny, R.E.; Allen, P.D.; Grossman, W.; Gwathmey, J.K. Abnormal intracellular calcium handling, a major cause of systolic and diastolic dysfunction in ventricular myocardium from patients with heart failure. Circulation 1990, 81, 21-32.

5. Kiss, E.; Ball, N.A.; Kranias, E.G.; Walsh, R.A. Differential changes in cardiac phospholamban and sarcoplasmic reticular $\mathrm{Ca}^{2+}$-ATPase protein levels. Effects on $\mathrm{Ca}^{2+}$ transport and mechanics in compensated pressure-overload hypertrophy and congestive heart failure. Circ. Res. 1995, 77, 759-764. [CrossRef] [PubMed]

6. Groschner, K.; Graier, W.F.; Romanin, C. Store-Operated CA2 Entry (SOCE) Pathway: Emerging Signaling Concepts in Human (Patho)Physiology; Springer-Verlag Wien: Vienna, Austria, 2017; ISBN 978-3-319-57732-6.

7. Montell, C.; Rubin, G.M. Molecular characterization of the Drosophila trp locus: A putative integral membrane protein required for phototransduction. Neuron 1989, 2, 1313-1323. [CrossRef]

8. Nilius, B. TRP channels in disease. Biochim. Biophys. Acta BBA Mol. Basis Dis. 2007, 1772, 805-812. [CrossRef]

9. Venkatachalam, K.; Montell, C. TRP channels. Annu. Rev. Biochem. 2007, 76, 387-417. [CrossRef]

10. Boulay, G.; Brown, D.M.; Qin, N.; Jiang, M.; Dietrich, A.; Zhu, M.X.; Chen, Z.; Birnbaumer, M.; Mikoshiba, K.; Birnbaumer, L. Modulation of $\mathrm{Ca}^{2+}$ entry by polypeptides of the inositol 1,4,5-trisphosphate receptor (IP3R) that bind transient receptor potential (TRP): Evidence for roles of TRP and IP3R in store depletion-activated $\mathrm{Ca}^{2+}$ entry. Proc. Natl. Acad. Sci. USA 1999, 96, 14955-14960. [CrossRef]

11. Schaefer, M.; Plant, T.D.; Obukhov, A.G.; Hofmann, T.; Gudermann, T.; Schultz, G. Receptor-mediated regulation of the nonselective cation channels TRPC4 and TRPC5. J. Biol. Chem. 2000, 275, 17517-17526. [CrossRef]

12. Shi, J.; Mori, E.; Mori, Y.; Mori, M.; Li, J.; Ito, Y.; Inoue, R. Multiple regulation by calcium of murine homologues of transient receptor potential proteins TRPC6 and TRPC7 expressed in HEK293 cells. J. Physiol. 2004, 561, 415-432. [CrossRef] [PubMed]

13. Yue, Z.; Xie, J.; Yu, A.S.; Stock, J.; Du, J.; Yue, L. Role of TRP channels in the cardiovascular system. Am. J. Physiol. Heart Circ. Physiol. 2015, 308, 157-182. [CrossRef] [PubMed]

14. Eder, P.; Molkentin, J.D. TRPC channels as effectors of cardiac hypertrophy. Circ. Res. 2011, 108, $265-272$. [CrossRef] [PubMed]

15. Bush, E.W.; Hood, D.B.; Papst, P.J.; Chapo, J.A.; Minobe, W.; Bristow, M.R.; Olson, E.N.; McKinsey, T.A. Canonical transient receptor potential channels promote cardiomyocyte hypertrophy through activation of calcineurin signaling. J. Biol. Chem. 2006, 281, 33487-33496. [CrossRef] [PubMed]

16. Nakayama, H.; Wilkin, B.J.; Bodi, I.; Molkentin, J.D. Calcineurin-dependent cardiac hypertrophy is activated by TRPC in the adult mouse heart. FASEB J. Off. Publ. Fed. Am. Soc. Exp. Biol. 2006, 20, 1660-1670.

17. Iwata, Y.; Ohtake, H.; Suzuki, O.; Matsuda, J.; Komamura, K.; Wakabayashi, S. Blockade of sarcolemmal TRPV2 accumulation inhibits progression of dilated cardiomyopathy. Cardiovasc. Res. 2013, 99, 760-768. [CrossRef] [PubMed]

18. Buckley, C.L.; Stokes, A.J. Mice lacking functional TRPV1 are protected from pressure overload cardiac hypertrophy. Channels 2011, 5, 367-374. [CrossRef] [PubMed]

19. Kuwahara, K.; Wang, Y.; McAnally, J.; Richardson, J.A.; Bassel-Duby, R.; Hill, J.A.; Olson, E.N. TRPC6 fulfills a calcineurin signaling circuit during pathologic cardiac remodeling. J. Clin. Investig. 2006, 116, 3114-3126. [CrossRef] [PubMed]

20. Morine, K.J.; Paruchuri, V.; Qiao, X.; Aronovitz, M.; Huggins, G.S.; DeNofrio, D.; Kiernan, M.S.; Karas, R.H.; Kapur, N.K. Endoglin selectively modulates transient receptor potential channel expression in left and right heart failure. Cardiovasc. Pathol. 2016, 25, 478-482. [CrossRef] 
21. Chronic Heart Failure in Adults: Diagnosis and Management Guidance and Guidelines NICE. Available online: https://www.nice.org.uk/guidance/ng106/chapter/recommendations\#heart-failure-withreduced-ejection-fraction (accessed on 15 November 2018).

22. Gees, M.; Colsoul, B.; Nilius, B. The role of transient receptor potential cation channels in $\mathrm{Ca}^{2+}$ signaling. Cold Spring Harb. Perspect. Biol. 2010, 2, a003962. [CrossRef]

23. Seth, M.; Zhang, Z.-S.; Mao, L.; Graham, V.; Burch, J.; Stiber, J.; Tsiokas, L.; Winn, M.; Abramowitz, J.; Rockman, H.A.; et al. TRPC1 channels are critical for hypertrophic signaling in the heart. Circ. Res. 2009, 105, 1023-1030. [CrossRef] [PubMed]

24. Ohba, T.; Watanabe, H.; Murakami, M.; Takahashi, Y.; Iino, K.; Kuromitsu, S.; Mori, Y.; Ono, K.; Iijima, T.; Ito, H. Upregulation of TRPC1 in the development of cardiac hypertrophy. J. Mol. Cell. Cardiol. 2007, 42, 498-507. [CrossRef] [PubMed]

25. Wu, X.; Eder, P.; Chang, B.; Molkentin, J.D. TRPC channels are necessary mediators of pathologic cardiac hypertrophy. Proc. Natl. Acad. Sci.USA 2010, 107, 7000-7005. [CrossRef]

26. Hofmann, T.; Schaefer, M.; Schultz, G.; Gudermann, T. Subunit composition of mammalian transient receptor potential channels in living cells. Proc. Natl. Acad. Sci. USA 2002, 99, 7461-7466. [CrossRef] [PubMed]

27. AL-Shawaf, E.; Naylor, J.; Taylor, H.; Riches, K.; Milligan, C.J.; O’Regan, D.; Porter, K.E.; Li, J.; Beech, D.J. Acute stimulation of calcium-permeable TRPC5-containing channels by oxidized phospholipids. Arter. Thromb. Vasc. Biol. 2010, 30, 1453-1459. [CrossRef] [PubMed]

28. Seo, K.; Rainer, P.P.; Lee, D.; Hao, S.; Bedja, D.; Birnbaumer, L.; Cingolani, O.H.; Kass, D.A. Hyperactive adverse mechanical-stress responses in dystrophic heart are coupled to TRPC6 and blocked by cGMP-PKG modulation. Circ. Res. 2014, 114, 823-832. [CrossRef] [PubMed]

29. Hofmann, T.; Chubanov, V.; Gudermann, T.; Montell, C. TRPM5 is a voltage-modulated and $\mathrm{Ca}^{2+}$-activated monovalent selective cation channel. Curr. Biol. 2003, 13, 1153-1158. [CrossRef]

30. Launay, P.; Fleig, A.; Perraud, A.-L.; Scharenberg, A.M.; Penner, R.; Kinet, J.-P. TRPM4 is a Ca ${ }^{2+}$-activated nonselective cation channel mediating cell membrane depolarization. Cell 2002, 109, 397-407. [CrossRef]

31. Nadler, M.J.S.; Hermosura, M.C.; Inabe, K.; Perraud, A.L.; Zhu, Q.; Stokes, A.J.; Kurosaki, T.; Kinet, J.P.; Penner, R.; Scharenberg, A.M.; et al. LTRPC7 is a Mg.ATP-regulated divalent cation channel required for cell viability. Nature 2001, 411, 590-595. [CrossRef]

32. Guinamard, R.; Demion, M.; Magaud, C.; Potreau, D.; Bois, P. Functional Expression of the TRPM4 cationic current in ventricular cardiomyocytes from spontaneously hypertensive rats. Hypertension 2006, 48, 587-594. [CrossRef]

33. Guinamard, R.; Bois, P. Involvement of transient receptor potential proteins in cardiac hypertrophy. Biochim. Biophys. Acta BBA Mol. Basis Dis. 2007, 1772, 885-894. [CrossRef] [PubMed]

34. Köster, O.F.; Szigeti, G.P.; Beuckelmann, D.J. Characterization of a $\left[\mathrm{Ca}^{2+}\right]_{\mathrm{i}}$-dependent current in human atrial and ventricular cardiomyocytes in the absence of $\mathrm{Na}^{+}$and $\mathrm{K}^{+}$. Cardiovasc. Res. 1999, 41, 175-187. [CrossRef]

35. Kecskés, M.; Jacobs, G.; Kerselaers, S.; Syam, N.; Menigoz, A.; Vangheluwe, P.; Freichel, M.; Flockerzi, V.; Voets, T.; Vennekens, R. The $\mathrm{Ca}^{2+}$-activated cation channel TRPM4 is a negative regulator of angiotensin II-induced cardiac hypertrophy. Basic Res. Cardiol. 2015, 110, 43. [CrossRef] [PubMed]

36. Du, J.; Xie, J.; Zhang, Z.; Tsujikawa, H.; Fusco, D.; Silverman, D.; Liang, B.; Yue, L. TRPM7-mediated Ca ${ }^{2+}$ signals confer fibrogenesis in human atrial fibrillation. Circ. Res. 2010, 106, 992-1003. [CrossRef] [PubMed]

37. Kanzaki, M.; Zhang, Y.Q.; Mashima, H.; Li, L.; Shibata, H.; Kojima, I. Translocation of a calcium-permeable cation channel induced by insulin-like growth factor-I. Nat. Cell Biol. 1999, 1, 165-170. [CrossRef]

38. Iwata, Y.; Katanosaka, Y.; Arai, Y.; Komamura, K.; Miyatake, K.; Shigekawa, M. A novel mechanism of myocyte degeneration involving the $\mathrm{Ca}^{2+}$-permeable growth factor-regulated channel. J. Cell Biol. 2003, 161, 957-967. [CrossRef] [PubMed]

39. Katanosaka, Y.; Iwasaki, K.; Ujihara, Y.; Takatsu, S.; Nishitsuji, K.; Kanagawa, M.; Sudo, A.; Toda, T.; Katanosaka, K.; Mohri, S.; et al. TRPV2 is critical for the maintenance of cardiac structure and function in mice. Nat. Commun. 2014, 5, 3932. [CrossRef] [PubMed]

40. Numaga-Tomita, T.; Oda, S.; Nishiyama, K.; Tanaka, T.; Nishimura, A.; Nishida, M. TRPC channels in exercise-mimetic therapy. Pflug. Arch. 2019, 471, 507-517. [CrossRef] [PubMed]

(C) 2019 by the authors. Licensee MDPI, Basel, Switzerland. This article is an open access article distributed under the terms and conditions of the Creative Commons Attribution (CC BY) license (http://creativecommons.org/licenses/by/4.0/). 